\title{
Price-Setting Combinatorial Auctions for Coordination and Control of Manufacturing Multiagent Systems: Updating Prices Methods
}

\author{
Juan José Lavios Villahoz ${ }^{1}$, Ricardo del Olmo Martínez ${ }^{1}$, \\ and Alberto Arauzo Arauzo ${ }^{2}$ \\ ${ }^{1}$ INSISOC, Escuela Politécnica Superior, Universidad de Burgos \\ Avda, Cantabria s/n, 09006, Burgos, Spain \\ \{jjlavios, rdelolmo\} @ubu.es \\ ${ }^{2}$ INSISOC, ETSII Universidad de Valladolid \\ $\mathrm{P}^{\mathrm{o}}$ del Cauce s/n, 47011 Valladolid, Spain \\ arauzo@insisoc.org
}

\begin{abstract}
Combinatorial auctions are used as a distributed coordination mechanism in Multiagent Systems. The use of combinatorial auctions as negotiation and coordination mechanism is especially appropriate in systems with interdependencies and complementarities such as manufacturing scheduling systems. In this work we review some updating price mechanisms for combinatorial auctions based on the Lagrangian Relaxation Method. We focus our research to solve the optimization scheduling problem in the shop floor, taking into account the objectives of resource allocation in dynamic environments, i.e. -robustness, stability, adaptability, and efficiency-.
\end{abstract}

Keywords: Multiagent Systems, Combinatorial Auction, Lagrangian Relaxation.

\section{Introduction}

Scheduling is a decision-making problem devoted to allocate resources on tasks to optimize one or several objectives. Manufacturing and production systems are some of the most known fields of application of this problem. In this context, operations of a given production process are considered as tasks and the different machines in a workshop are considered the resources [1]. This problem is characterized by its highly combinatorial and dynamic nature and its practical interest for industrial applications [2].

Multiagent Systems (MAS) have been proved to be an appropriate paradigm to model complex systems and they constitute a useful framework to define distributed decision-making processes. In this specific domain, agents are used to encapsulate physical and logical entities or even autonomous functionalities of the production systems. The ability of MAS to perceive and react to changes in their environment justifies their use as adaptive systems in manufacturing (some recent reviews in the field can be found in [3], [4] or [5]. 


\section{Market Based Coordination Mechanisms and Combinatorial Auctions}

Distributed decision making through MAS are considered an alternative to centralized scheduling systems. They facilitate to take into account local objectives, preferences and constraints of each resource in the decision making process [6]. Combination of individual problem-solving and coordination/negotiation schemes is one of the research challenges in this area [3].

Market based allocation mechanisms are one of the most active branches of decentralized scheduling research. The underlying idea is to allocate resources among tasks by designing an ad-hoc market and setting prices iteratively to find the equilibrium. The process involves the creation of a production schedule based on the prices emerging from the bids sent by tasks. The goal is to design a bid structure and a selling mechanism that leads to an optimal scheduling of resources on a shop floor.

According to [7], the main features of a decentralized scheduling problem are: First, each individual decision-maker follow its own objectives, but coordination will be enabled by prices. Second, decision-makers may have their own private information, such as their valuations of objects, and have no access to the private information of others (objectives, preferences and constraints). Third, complex calculations are distributed among the participating agents, so that the problem is divided into several easier problems which can be solved in parallel, and hence calculations can be sped up. Fourth, the communication overhead is low since it is limited to the exchange of bids and prices between agents and the market mechanism.

Combinatorial auctions have been used as a market mechanism to create schedules. One of the earliest works in this area has been developed by [8]. Combinatorial auction is a type of auction in which participants can place bids on combinations of items rather than just individual items or continuous quantities. Economic efficiency is enhanced if bidders are allowed to bid on bundles of different items when there are complementarities between the different items, [9]. The use of combinatorial auctions as negotiation and coordination mechanism is appropriate in problems in which the value of interdependent items needs to be considered.

Manufacturing scheduling problem presents complementarities. For instance, jobs are required to bid for continuous time slots on a resource (non-preemption constraints) and each job is required to participate in multiple auctions for its different operations, however all these operations have precedence constraints. We use a combinatorial auction-based mechanism to coordinate agents.

Combinatorial auctions can be divided into single round and iterative combinatorial auctions. In single round auctions participants send their valuations over the combinations of products just one time and the auctioneer allocate the items in such a way that the global objective is maximized. On the contrary, in iterative combinatorial auctions prices are fixed after multiple rounds. There are some advantages of using iterative combinatorial auctions instead of single round ones. First, participants do not have to make bids over the set of all possible combinations of bids. Second, participants reveal in each iteration their private information and preferences. Third, iterative auctions are well-suited for dynamic environments (e.g. manufacturing environments) where participants and items get in and out in different moments. 
There are two kinds of iterative combinatorial auctions: quantity-setting and pricesetting. In the fist one, bidders send their valuation over every possible combination of the items sold. The auctioneer makes a provisional allocation that depends on the submitted prices. Bidders adjust the prices at every iteration. In the second one, price-setting, (or demand query) auctioneer sets the prices for each of the items of the auction. Bidders submit the bundle of items they want to get at the given price. Auctioneer adapts the prices to balance the supply and demand [9].

\section{Combinatorial Auctions and Lagrange Relaxation Techniques}

The updating methods used in the Lagrangian Relaxation Method to solve the dual problem can be used to update prices in iterative combinatorial auctions, since they share the same structure in their protocols [10]. Auction protocols can be implemented in a non-adaptive standard Walrasian fashion, or as an adaptive tâtonement (price adjustment process). The subgradient optimization can be viewed as a particular version of the tâtonement proccess. While the subgradient optimization tries to penalize infeasibility of dualized constraints and the auctioneer updates the resource prices as to discourage conflicts on the demanded objects, the subgradient algorithm adjusts the prices proportional to the amount of infeasibility and the auction algorithm updates the prices proportional to the excess of demand [6]. Lagrangian relaxation technique relaxes the complex constraints of an optimization problem (i.e. those constraints which make the problem hard to solve), making the problem easier to optimize. The group of relaxed constraints is incorporated to the objective function in such a way that the restrictions that are not fulfilled are penalized. The new objective function is called Lagrangian function. The Lagrangian problem solution is always lower or equal to the solution of the original problem [11]. The iterative process will approach the solution of the Lagrangian problem to the solution of the original problem.

\section{Price Setting Iterative Combinatorial Auction}

The task scheduling problem can be modeled as an auction where time horizon is divided into slots that are sold through the auction. Tasks participate in the auction as bidders, trying to get the time slots of the resources that they need to perform the operation [10](See Fig. 1). We will relax the constraints that relies on variables belonging to different jobs. Our aim is to divide a complex problem into several easier ones. If we eliminate the restriction of capacity of the resources, we will be able to split the problem into $\mathrm{N}$ job subproblems. The mechanism will follow the main principles of distributed systems since the relevant information of the bidders (i.e. due date of the jobs, penalty for the delays, resources needed) will be hidden to the rest of agents [12]. None of the agents knows which other agents belong to the system nor what are the goals of those agents. Prices show the preferences of other agents, providing information to the agent to act consequently. This mechanism have been used to solve different kind of task scheduling problem, e.g. job shop problem [13], [14], flow shop problem [15], or project management [16], [17]. 


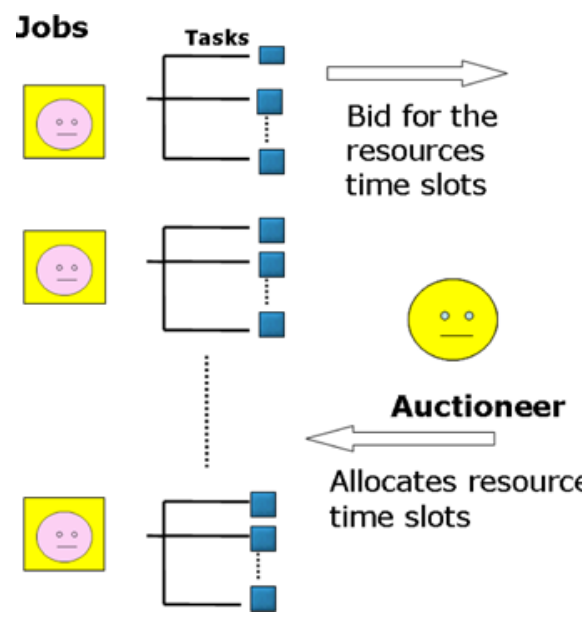

\section{Pool of resources}

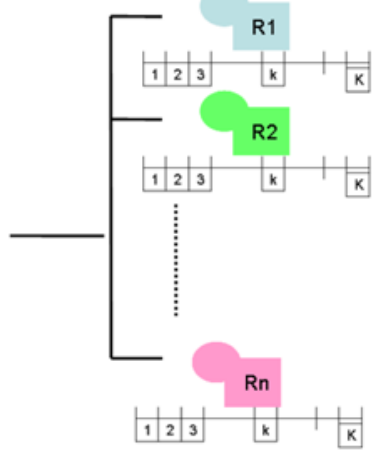

Fig. 1. Auction-based scheduling mechanism

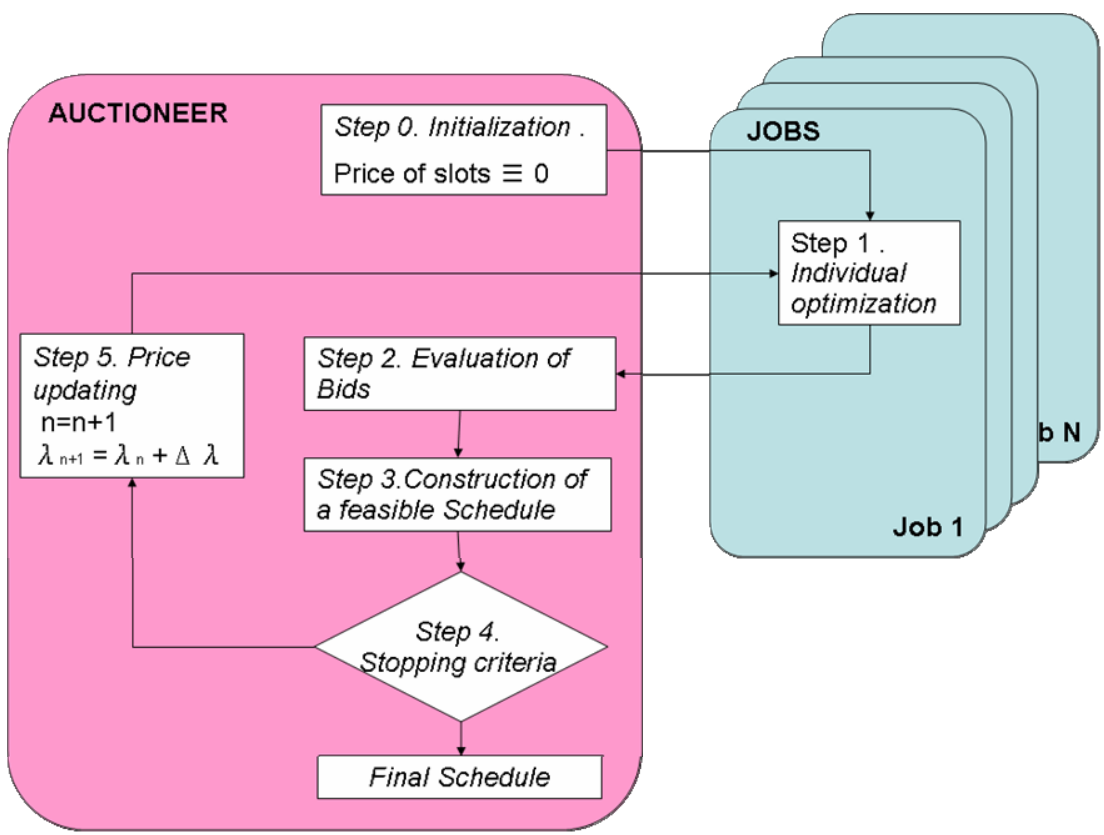

Fig. 2. Price-setting iterative combinatorial auction

It can be described as follows: There exists a central pool of resources (Resourceagent). Each resource has different abilities. The planning horizon of the resource is divided into time slots. These time slots are sold in an auction. Several jobs have to be completed (Job-agents). The jobs need to get the necessary resources to be finished before their due date. Job-agents bid for the time slot of the resources. They try to 
minimize their cost function. An agent acts as a central node (Auctioneer-agent). The bids sent by Job-agents are received by the Auctioneer-agent, who will update the prices of the slots. Once known the new prices, the Job-agents will remake their bids. This iterative process continues until prices are stabilized or a stop condition is fulfilled. (See fig. 2)

Job-agents are price-takers in the model (See fig. 3). Price is fixed by an iterative process. The prices of the slots are raised or lowered by a walrasian mechanism. The auctioneer compares the demand over a time slot of a resource with the capacity of the resource in this time slot. if there is an excess demand, the auctioneer raises prices. On the other hand, if there is an excess of capacity he lowers them.

$$
\lambda^{n+1}=\lambda^{n}+\Delta \lambda
$$

There are many ways to update prices (e.g. constant increase or decrease of prices, proportional to the demand, proportional to the excess of demand).

\section{Updating Prices Methods}

Subgradient method [18], [11], [19]: It is the method most commonly used to solve the relaxed (dual) problem. Each bidder sends the bundle of time slots that maximizes its utility. Auctioneer computes the excess demand (subgradient) and updates time slots prices along the subgradient. A lower bound of the optimal scheduling cost can be obtained from the dual problem resolution process. Although it is used by many practitioners for its simplicity and low computational overhead, it has many drawbacks: Multipliers zigzag along ridges (intersection of several facets) of the dual function. It suffers from slow convergence, and it can be difficult to solve all the suproblems in big size problems.

Aiming to improve the performance of the subgradient method, [20] developed the conjugate gradient method, also used in [21], [22] or [23] by following a modified gradient step direction that is calculated as a linear combination of the actual vector of excess demand and the precedent modified gradient step direction. It reduces zigzagging with respect to subgradient method.

The surrogate gradient method [24], [25] is based on the idea that it is not necessary to get the best directions to reach the optimum of the dual function. Good directions can be obtained with much less effort. The bundles of time slots sent by the bidders do not have to maximize its utility; they just have to improve the utility of the bidder with respect to the previous bids at the given prices. This method is useful for large problems having many complicated subproblems. The main disadvantage of this method is that the surrogate dual cost is not always a lower bound to the optimal scheduling cost.

The interleaved subgradient is a special case of the surrogate method [26], [13], [24]. Only one subproblem per iteration is solved to obtain a direction and to update the multipliers. The method is called random interleaved subgradient if this process is done in a non predefined order. This idea is attractive for real dynamic environments, where real time dynamics have to be considered. The directions obtained are also smooth for large problems, leading to better performance. 
The fuzzy subgradient [27] aims to make use of all the information generated in the job sub-problem resolution. It takes not only optimal solutions of the job subproblems, but also near optimal solutions combining them to generate "fuzzy gradients". This method reduces the solution zigzagging without much additional computational requirements.

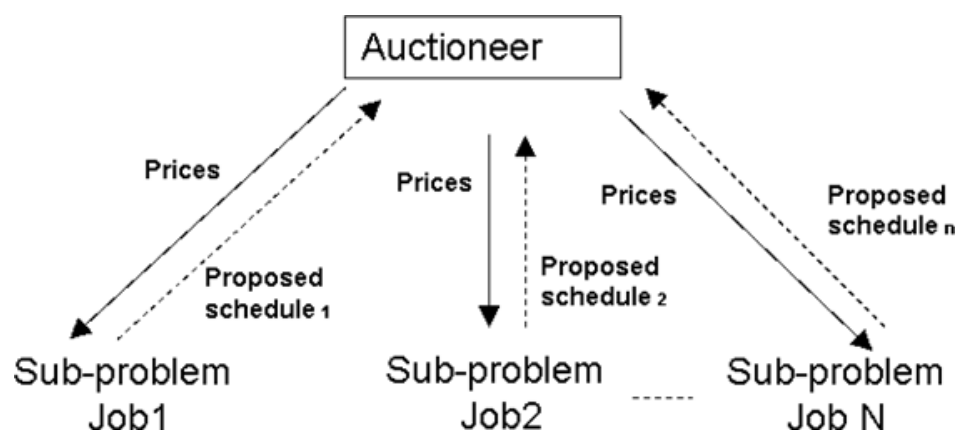

Fig. 3. Problem-solution schema

\section{Research Line and Future Work}

We search an updating price method for combinatorial auction that meets the requirements of scheduling manufacturing systems in dynamic environments, i.e. they are intended to offer robustness, stability, adaptability, and efficient use of available resources through a modular and distributed design [5]. To the best of our knowledge, currently there is no work which compares the updating prices methods of combinatorial auctions for Job Shop problem.. In our work we will use a specific problem as Job Shop scheduling problem, but we want to extend the conclusions that we will obtain to other problems of the same nature. Our objective is to compare the different methods of updating prices based on those that update the Lagrangian problem.

We will define and implement a distributed task scheduling system based on the Job Shop Problem [1]. We want to compare the different methods of updating prices using as criteria convergence, stability and other points to study in a real case implementation as asynchronous computation. We will use different benchmark as we can find in [28] and the modifications suggested in [29].

\section{Acknowledgements}

This work has been partially supported Caja de Burgos through Projects 2009/ 00199/001 and 2009/00148/001.

\section{References}

1. Pinedo, E.P.M.L.: Scheduling, 2nd edn. Springer, New York (2008)

2. Shen, W.: Distributed manufacturing scheduling using intelligent agents. Intelligent Systems 17(1), 88-94 (2002) 
3. Shen, W., et al.: Applications of agent-based systems in intelligent manufacturing: An updated review. Advanced Engineering Informatics 20(4), 415-431 (2006)

4. Lee, J.-H., Kim, C.-O.: Multi-agent systems applications in manufacturing systems and supply chain management: a review paper. International Journal of Production Research 46(1), 233-265 (2008)

5. Ouelhadj, D., Petrovic, S.: A survey of dynamic scheduling in manufacturing systems. Journal of Scheduling 12(4), 417-431 (2009)

6. Kutanoglu, E., Wu, S.D.: On combinatorial auction and Lagrangean relaxation for distributed resource scheduling. IIE Transactions 31(9), 813-826 (1999)

7. Wellman, M.P.: A Market-Oriented Programming Environment and its Application to Distributed Multicommodity Flow Problems. Journal of Artificial Intelligence Research 1, 123 (1993)

8. Rassenti, S., Smith, V., Bulfin, R.: A Combinatorial Auction Mechanism for Airport Time Slot Allocation. The Bell Journal of Economics 13(2), 402-417 (1982)

9. de Vries, S., Vohra, R.V.: Combinatorial Auctions: A Survey. Informs Journal on Computing 15(3), 284-309 (2003)

10. Dewan, P., Joshi, S.: Auction-based distributed scheduling in a dynamic job shop environment. International Journal of Production Research 40(5), 1173-1191 (2002)

11. Fisher, M.L.: The Lagrangian Relaxation Method for Solving Integer Programming Problems. Management Science 50(12 Suppl.), 1861-1871 (2004)

12. Duffie, N.A.: Synthesis of heterarchical manufacturing systems. Comput. Ind. 14(1-3), 167-174 (1990)

13. Kaskavelis, C.A., Caramanis, M.C.: Efficient Lagrangian relaxation algorithms for industry size job-shop scheduling problems. IIE Transactions 30(11), 1085-1097 (1998)

14. Sun, T., Luh, P., Liu, M.: Lagrangian relaxation for complex job shop scheduling. In: Proceedings 2006 IEEE International Conference on En Robotics and Automation, ICRA 2006, pp. 1432-1437 (2006)

15. Tang, L., Xuan, H., Liu, J.: A new Lagrangian relaxation algorithm for hybrid flowshop scheduling to minimize total weighted completion time. Computers \& Operations Research 33(11), 3344-3359 (2006)

16. Ni, M., Luh, P., Moser, B.: An Optimization-Based Approach for Design Project Scheduling. IEEE Transactions on Automation Science and Engineering 5(3), 394-406 (2008)

17. Arauzo, J., et al.: Gestión eficiente de carteras de proyectos. Propuesta de un sistema inteligente de soporte a la decisión para oficinas técnicas y empresas consultoras= efficient projet porfolio management. And intelligent decision system for engineering and consultancy firms. Dyna 84(9), 761-772 (2009)

18. Geoffrion, A.M.: Lagrangean relaxation for integer programming. In: En Approaches to Integer Programming, pp. 82-114 (1974)

19. Guignard, M.: Lagrangean relaxation. TOP 11(2), 151-200 (2003)

20. Camerini, P.M., Fratta, L., Maffioli, F.: On improving relaxation methods by modified gradient techniques. En Nondifferentiable Optimization, 26-34 (1975)

21. Crowder, H.: Computational Improvements for Subgradient Optimization. In: Symposia Mathematica, pp. 357-372. Academic Press, New York (1976)

22. Brännlund, U.: A generalized subgradient method with relaxation step. Mathematical Programming 71(2), 207-219 (1995)

23. Wang, J., et al.: An optimization-based algorithm for job shop scheduling. SADHANA 22, 241-256 (1997) 
24. Zhao, X., Luh, P., Wang, J.: The surrogate gradient algorithm for Lagrangian relaxation method. In: Proceedings of the 36th IEEE Conference on Decision and Control, vol. 1, pp. 305-310 (1997)

25. Chen, H., Luh, P.: An alternative framework to Lagrangian relaxation approach for job shop scheduling. European Journal of Operational Research 149(3), 499-512 (2003)

26. Zhao, X., Luh, P.B., Wang, J.: Surrogate Gradient Algorithm for Lagrangian Relaxation. Journal of Optimization Theory and Applications 100(3), 699-712 (1999)

27. Zhao, X., Luh, P.: Fuzzy gradient method in Lagrangian relaxation for integer programming problems. In: Proceedings of the 37th IEEE Conference on En Decision and Control, vol. 3, pp. 3372-3377 (1998)

28. Demirkol, E., Mehta, S., Uzsoy, R.: Benchmarks for shop scheduling problems. European Journal of Operational Research 109(1), 137-141 (1998)

29. Kreipl, S.: A large step random walk for minimizing total weighted tardiness in a job shop. Journal of Scheduling 3(3), 125-138 (2000) 